\title{
Computer-Aided transoral partial mandibulectomy and reconstruction: Case Report
}

\author{
Christopher Liangcheng Chen ${ }^{1}$, Yan-Chi Chen ${ }^{1}$ and Michael Yuanchien Chen ${ }^{1,2 *}$ \\ ${ }^{1}$ Division of Oral \& Maxillofacial Surgery, Dental Department, China Medical University Hospital, Taichung, Taiwan \\ ${ }^{2}$ Dental College, China Medical University, Taichung, Taiwan
}

\begin{abstract}
Virtual planning software and three-dimensional printing technology have improved the operation time and surgical accuracy. We performed partial mandibulectomy and reconstruction via the intraoral approach with relatively acceptable clinical results in two patients with ameloblastoma. Preoperative preparations were full skull computed tomography, virtual surgical cuttings, pre-bent load-sharing titanium plates that conformed to the additive stereolithographic models, and complex computer-aided design/computer-assisted manufacturing surgical guide for intraoperative screw placement and plate fixation. Procedures were performed transorally, but two tiny stab skin incisions were made on the unilateral posterior cheek for transbuccal screw fixation on proximal segments. Bony defects due to ameloblastoma were created by load-sharing plates and autogenous iliac bone grafts plus platelet rich plasma/fibrin followed by 6-week intermaxillary fixation. Transplanted autogenous bone grafts consolidated uneventfully, occlusion remained well and stable with intact facial profile, frontal symmetry, and inconspicuous incision marks on posterior cheeks. In selected cases, we resected huge pathologic lesions of the mandible and immediate reconstruction through a relatively conservative approach, attaining less donor site morbidities, reasonable functional and esthetic outcomes, and maintenance of adequate life quality. The perfusion status of the soft tissue matrix around the affected bone segment helps determine whether to adopt this protocol.
\end{abstract}

\section{Introduction}

Mandibular ameloblastoma is a common odontogenic tumor with incidence of approximately $9 \%-11 \%$ of odontogenic tumors [1]. It is a locally aggressive and disfiguring oral cavity tumor, and surgical management is the mainstay of treatment. Conservative treatment like enucleation and curettage is sometimes suspended because of its high recurrence rates. Contrastingly, segmental mandibulectomy for mandibular ameloblastoma have lower recurrence rates, but reconstruction remains a challenge for appearance maintenance, functional restoration, etc [2].

Esthetic outcomes have been an important goal of mandibular reconstruction after excision of jawbone tumors. The gold standard advocated by some authors for mandibulectomy and reconstruction might be osteocutaneous free tissue transfer with titanium plate fixation. However, complications such as facial nerve damage, surgical wound over the facial region, and larger donor site still existed. Operation via the transoral approach minimizes facial incisions and preserves the perioral musculature, achieving an esthetic and functional result $[3,4]$.

This case series report demonstrates that cautious case selection for transoral resection and reconstruction based on computer-aided design/computer-assisted manufacturing (CAD/CAM) is a different option to meet patient's high demands for esthetic outcomes.

\section{Case Descriptions}

Two patients underwent mandibulectomy and reconstruction in our hospital after diagnosis of ameloblastoma. 3D customized models and surgical guide were prefabricated using 3D computed tomography (CT) data, and titanium plates were bent before the operation. Autogenous iliac bone graft was applied. Surgery was performed via the intraoral approach; a single tiny incision $(1 \mathrm{~cm})$ was made over the facial skin for screw fixations.

\section{Case 1}

A 46-year-old female experienced expansile bone lesion at her right mandibular body angle with the involvement of the right mandibular canal leading to paresthesia of the right chin and lower lip.

\section{Patient's skull reference model}

The data files of cone beam CT (CBCT) were imported and segmented to create a patient-specific virtual model that would be used for planning. As reference for the fitting of the surgical guide, a 3D model of the patient's mandible was printed from the ${ }^{*}$.stl data of the patient's skeletal anatomy (Connex3 Objet500 [Stratasys, Eden Prairie, MN, USA]). The model was printed with a layer thickness of $0.030 \mathrm{~mm}$ using a printer jet with layers of curable liquid photopolymer providing strength and accuracy. With the patient's mandible reference model, we pre-bent the titanium plate (1.5 synthes) and fitted it smoothly to the mandible model.

\section{Virtual planning and surgical guide design}

Titanium plate fixated with the patient's mandible model was sent to the 3D printing center (China Medical University). Based on the

${ }^{*}$ Correspondence to: Michael Yuanchien Chen, Division of Oral \& Maxillofacial Surgery, Dental Department, China Medical University Hospital, Taichung, Taiwan, Dental College, China Medical University, Taichung, Taiwan, Tel: +886928182949; Fax: +886-04- 22037746; E-mail: mychen@mail.cmuh.org.tw

Key words: conservative treatment, intraoral approach, mandible 3D-model, mandibular ameloblastoma, mandibulectomy, reconstruction, surgical guide, titanium plate, transoral approach, virtual surgical planning

Received: July 23, 2019; Accepted: July 30, 2019; Published: August 02, 2019 
planned titanium plate position, surgical guide designs were virtually designed and ${ }^{*}$.stl files were generated for printing.

Seven monocortical screws were positioned along the mandible model, i.e., three on the proximal segments and four on the distal segments. Four smaller holes were used to lock the fixation screws with the occlusal surface to fixate the surgical guide. The narrow cuts over the guides were the reference for the resection of lesions, so that could we accurately complete the bone cut according to our design.

Access holes at $2 \mathrm{~mm}$ high were designed at the posterior sites to guide drilling to achieve appropriate angulation with the help of trocar. Boolean subtraction was used to define the access holes for drill guidance. A Boolean operation was also used to create the bone fitting surface of the guide. The final design was then exported as an *.stl file for printing.

\section{Case 2}

\section{Clinical report}

A 26-year-old female had an expansile bone lesion at her right mandibular body angle without neurosensory disturbance of the right inferior alveolar nerve. Histopathological reports revealed the lesion as ameloblastoma. CT images confirmed the cortical expansion and perforation on the buccal and lingual aspect of the tumor.

The patient's skull reference model and virtual planning and surgical guide design were acquired similar to that in Case 1 (Table 1 and Figure 1).

\section{Results}

Using the same protocols, successful clinical outcomes were achieved in both the patients. Postoperative clinical images showed good facial symmetry, stable occlusion, and invisible facial surgical wound. Both the patients had acceptable occlusal force.

At 2-year follow-up, both the patients showed good wound healing, and the panoramic image showed bone regeneration over the segmental mandibulectomy region, but no recurrent lesions. Both the patients denied any pain or discomfort (Figure 2). The posterior-anterior and lateral cephalometric films demonstrated good mandible symmetry and good mandible contour.

\section{Case 1}

The total operation time was 6 hours from the start of iliac bone graft harvesting until the end of mandibular reconstruction. Comparison between the preoperative and 2-year follow-up images show that the patient maintained her facial profile

The stab incision over the right facial region is not found even at early follow-up at 6 months.

Although teeth were missing over the right posterior region, the patient maintained quite acceptable occlusion after the surgery.

Postoperative panoramic image showed the distribution of the iliac bone graft at the surgical region, while at 2-year follow-up, good bone healing and substantial regeneration were obtained. Besides, no recurrent lesion was found.

\section{Case 2}

Cases 1 and 2 had similar surgical procedures and results (Figure 2); some of the differences between the two cases have been outlined in Table 1.

\section{Discussion}

Recently, with the help of CAD/CAM technology, the accuracy of the surgery has substantially improved [5]. Although the accuracy should be calculated statistically, which is not yet presented in this study, facial appearance was maintained. As there was no intraoperative reconstruction bending, the operative time was shortened $[6,7]$.

Two different designs of the surgical guide were used in these patients. In Case 1, a one-piece surgical guide was designed simply for better rigidity. However, in Case 2, this design met some difficulties
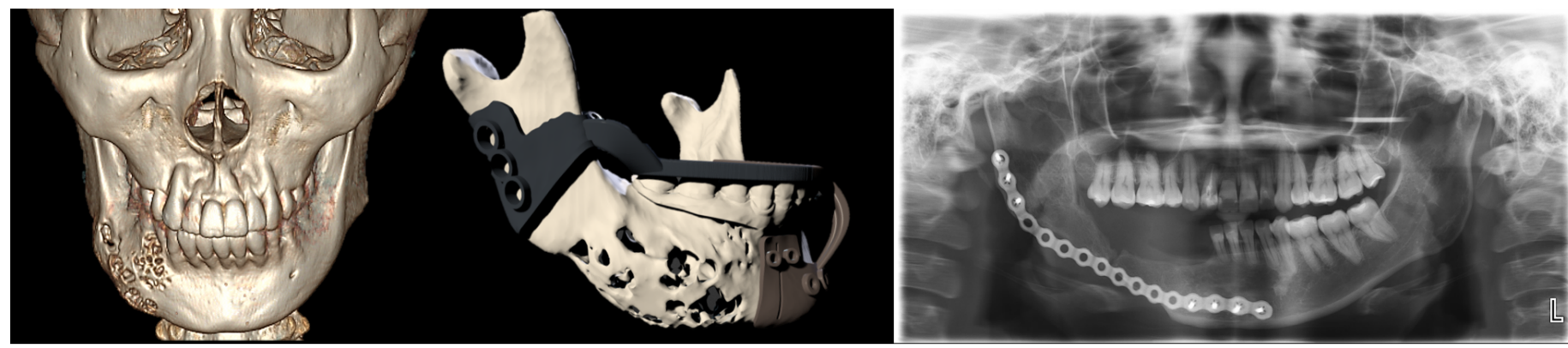

Figure 1. Cone beam CT (CBCT) scan data and surgical guide design for Case 2
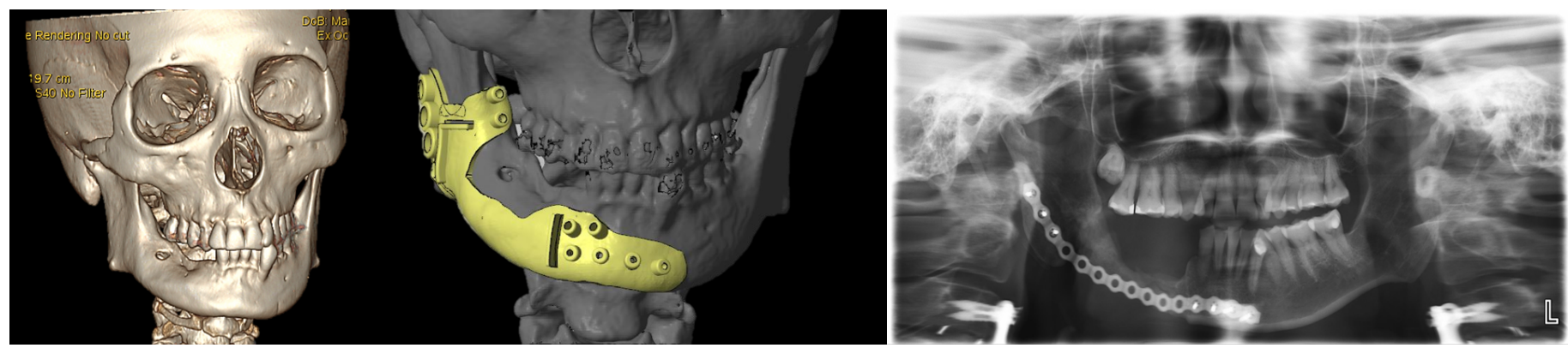

Figure 2. The 2-year follow-up panoramic film showed no recurrent lesions. There was good bone healing and substantial regeneration 
Table 1. Differences in clinical data and postoperative results between Case 1 and Case 2

\begin{tabular}{|l|l|l|}
\hline \multicolumn{2}{|l|}{ Case 1 } & Case 2 \\
\hline Clinical data & \multicolumn{2}{l|}{} \\
\hline Age (years) & 46 & 26 \\
\hline Sex & Female & Female \\
\hline Reconstruction plate & 1.5 synthes & 1.5 synthes \\
\hline Jaw classification & Right side & Right side \\
\hline Grafting materials & Autogenous iliac bone graft & Autogenous iliac bone graft \\
\hline Postoperative complication & nil & nil \\
\hline Facial scar & Invisible & Invisible \\
\hline CBCT scan voxel size & $\begin{array}{l}\text { (.625 mm } \times 0.625 \mathrm{~mm} \times \\
0.625 \mathrm{~mm}\end{array}$ & $\begin{array}{l}0.625 \mathrm{~mm} \times 0.625 \mathrm{~mm} \times \\
0.625 \mathrm{~mm}\end{array}$ \\
\hline Reference model material & Acrylic polymer & Acrylic polymer \\
\hline Postoperative results & & \\
\hline Operation time & 6 hours & 5 hours \\
\hline Stab incision & Invisible & Invisible \\
\hline Symmetry of the mandible & Yes & Yes \\
\hline Occlusion & Acceptable & Acceptable \\
\hline $\begin{array}{l}\text { Postoperative panoramic } \\
\text { follow-up of iliac bone graft } \\
\text { regeneration }\end{array}$ & Yes & $\begin{array}{l}\text { Yes } \\
\text { (Figure 1) }\end{array}$ \\
\hline Infection sign over mucosa & Nil & Nil \\
\hline & &
\end{tabular}

CBCT: cone beam computed tomography

owing to lesion swelling and improper attachment of stent to the tissue in the surgical region. The two-piece surgical guide was then designed for Case 2 (Figure 1).

Larger surgical wound of the donor site, long and visible facial scar, and increasing risk of facial nerve injury are inevitable in mandibulectomy and reconstruction via the extraoral approach $[8,9]$. Since then, the transoral approach not only provides good clinical results but also maintains patients' esthetics ideally. Moreover, no risk of facial nerve injury and smaller donor site are advantages of the transoral approach [10].

In addition to the methods used in the above two cases, benign tumors can be dealt with other methods considering the following criteria:

First, it must be a nonmalignant lesion. Wide excision would be inevitable leading to difficulty in primary wound closure. Second, there should be no active signs of infection; otherwise, failure of bone grafting is expected. Third, soft tissue including both skin and mucosa must be intact to allow successful surgical wound closure and bone grafting. Fourth, the remaining mandibular bone must have enough place for fixation. Fifth, patients with high esthetic demands might benefit from this approach, since no scar was visible after the operation.

With the limited number of cases, some preliminary conclusions could be made. $3 \mathrm{D}$ virtual planning not only decreased the operation time but also increased the accuracy of fixation. The intraoral approach provided pleasing esthetics outcome for patients who care about the facial scar. Good clinical result was achieved and maintained through 2 years after the operation.

\section{Acknowledgments}

This research was supported by China Medical University Hospital. We thank our colleagues from China Medical University Hospital who provided useful insights and expertise that greatly assisted the research, although they may not agree with all of the interpretations/conclusions of this manuscript.

We thankChia-Hui Yang (Case Manager, Ever Young BioDimension) and Min Chia Tsai (Radiologist, China Medical University Hospital) for their assistance in providing detailed data of 3D technology and CBCT. We would like to show our gratitude toward Lo Wen Fu, Doctor and teaching promoter, China Medical University Hospital, and Pei Ying Chang, Dean, Chang's Oral Surgery Clinic, for their comments that enhanced the manuscript.

We would like to thank Enago (www.enago.jp) for the English language review.

\section{Grant support}

\section{None}

\section{Conflicts of interest}

The authors declare no conflicts of interest.

\section{Ethics}

This study was approved by Research Ethics committee of China Medical University and Hospital (CMUH108-REC-001). Written informed consent was obtained from all patients.

\section{References}

1. Masthan KM, Anitha N, Krupaa J, Manikkam S (2015) Ameloblastoma. J Pharm Bioallied Sci 7: S167-S170. [Crossref]

2. Bak M, Jacobson AS, Buchbinder D, Urken ML (2010) Contemporary reconstruction of the mandible. Oral Oncol 46: 71-76. [Crossref]

3. Matsuo A, Chiba H, Toyoda J, Abukawa H, Fujikawa K, et al. (2011) Mandibular reconstruction using a tray with particulate cancellous bone and marrow and plateletrich plasma by an intraoral approach. J Oral Maxillofac Surg 69: 1807-1814.

4. Diaz-Carandell A, Agut-Busquet E, Molina-Montes J, Escuder-LaTorre O (2014) Intraoral approach and stereolithographic-guided large mandibular reconstruction with fibula free flap. Plast Reconstr Surg Glob Open 2: e199.

5. Hertog D, Bloemena E, Aartman IH, van-der-Waal I (2012) Histopathology of ameloblastoma of the jaws; some critical observations based on a 40 years single institution experience. Med Oral Patol Oral Cir Bucal 17: e76-e82.

6. Hammarfjord O, Roslund J, Abrahamsson P, Nilsson P, Thor A, et al. (2013) Surgical treatment of recurring ameloblastoma, are there options? Br J Oral Maxillofac Surg 51: 762-766.

7. de Paulo LF, Oliveira MT, Rodrigues ÁR, Zanetta-Barbosa D (2015) Treatment of an extensive unicystic ameloblastoma in a 7-year-old child: the best approach? Br J Oral Maxillofac Surg 53: 292-294.

8. Reichart PA, Philipsen HP, Sonner S (1995) Ameloblastoma: biological profile of 3677 cases. Eur J Cancer B Oral Oncol 31B: 86-99.

9. Robinson L, Martinez MG (1977) Unicystic ameloblastoma: a prognostically distinct entity. Cancer 40: 2278-2285.

10. McClary AC, West RB, McClary AC, Pollack JR, Fischbein NJ, et al. (2016) Ameloblastoma: a clinical review and trends in management. Eur Arch Otorhinolaryngol 273: 1649-1661. [Crossref]

Copyright: (C2019 Chen CL. This is an open-access article distributed under the terms of the Creative Commons Attribution License, which permits unrestricted use, distribution, and reproduction in any medium, provided the original author and source are credited. 